\title{
Editorial review of HPLSE special issue on laboratory astrophysics
}

\author{
Francisco Suzuki-Vidal ${ }^{1}$, Yutong $\mathrm{Li}^{2}$, Carolyn Kuranz ${ }^{3}$, and Colin Danson ${ }^{1,4}$ \\ ${ }^{1}$ Blackett Laboratory, Imperial College London, London SW7 2AZ, UK \\ ${ }^{2}$ Institute of Physics, Chinese Academy of Sciences, Beijing 100190, China \\ ${ }^{3}$ Climate and Space Science and Engineering, University of Michigan, Ann Arbor, MI 48109, USA \\ ${ }^{4} A W E$ plc, Aldermaston, Reading RG7 4PR, UK \\ (Received 7 December 2018; accepted 15 January 2019)
}

In 2018 the journal High Power Laser Science and Engineering produced a Special Issue on Laboratory Astrophysics. The scope of the special issue was to span the latest research and reviews on the following topics related to laboratory astrophysics and related phenomena. The topics invited for inclusion were:

- collisionless shocks

- planetary formation dynamics and planetary interiors

- warm dense matter

- hydrodynamic and magnetohydrodynamic instabilities

- magnetic reconnection

- relativistic plasmas

- magnetic turbulence and magnetic amplification

- nuclear astrophysics

- radiative transfer and radiation hydrodynamics

- target design

- laser-based HED facilities

although this was not meant as an exhaustive list. As is usual with a special issue of this type Guest Editors were invited to lead in sourcing articles. These editors were: Francisco Suzuki-Vidal, Imperial College London, UK; Yutong Li, Institute of Physics, Chinese Academy of Sciences, China; and Carolyn Kuranz, University of Michigan, USA.

Correspondence to: F. Suzuki-Vidal, Blackett Laboratory, Imperial College London, London SW7 2AZ, UK. Email: f.suzuki@imperial.ac.uk
Laboratory astrophysics is a novel research area that aims at studying physical processes which are commonly found in astrophysical scenarios but rarely occur naturally on Earth. This field has seen a substantial growth in recent years due to the number of high-power laser facilities which have provided scope for new experimental configurations and research themes to be explored.

The papers published in this special issue can be broadly grouped within the following main applications to laboratory astrophysics: magnetized plasmas and collisionless shocks ${ }^{[1-5]}$, supersonic, radiative plasma flows and hydrodynamic instabilities ${ }^{[6-10]}$, experimental diagnostic development ${ }^{[11,12]}$, and direct astrophysical applications $^{[13]}$. In addition, there were three review papers ${ }^{[14-16]}$ that give an in-depth study of current applications in the field.

\section{Review papers}

This special issue has three review papers. An in-depth review of different experimental platforms for magneticreconnection studies, with emphasis on laser-driven magnetic reconnection (LDMR) for instance on Vulcan, OMEGA and SG-II, is presented in Ref. [15]. A thorough review of experimental methods for applications to warm dense matter (WDM) is presented in Ref. [16], whereas the various approaches to the generation of extreme magnetic fields $(B \sim 100$ s to $1000 \mathrm{~T}$ ) using capacitor-coil targets are presented in Ref. [14].

\section{Magnetized plasmas and collisionless shocks}

One of the novel applications of laboratory astrophysics in recent years has been the development of experimental platforms to introduce dynamically significant magnetic fields in laser-driven plasmas. This requires a unique combination of experimental platforms, for instance, a high-repetition laser and a large-scale magnetic field on $\mathrm{LAPD}^{[1]}$ to produce super-Alfvenic plasmas, or the use of pulse-power-driven 
MIFEDS coils with the OMEGA laser ${ }^{[2]}$ to study planetary magnetospheric processes. The formation of magnetized reverse plasmas to study accretion shocks on the LULI laser in France ${ }^{[3]}$ and experiments looking at the self-generation of electromagnetic fields via Weibel-type instabilities in counterpropagating plasmas at the SG-II laser in China ${ }^{[4]}$ are presented. A study of maser radiation from collisionless shocks is presented in Ref. [5].

\section{Supersonic, radiative plasma flows and hydrodynamic instabilities}

The study of laser-driven, supersonic plasma flows and radiative shocks has been one of the main application of laboratory astrophysics for more than a decade. This special issue presents new experimental configurations looking at the interaction of radiative shocks with a solid obstacle. An analytical model of the interaction for experiments done on the LULI laser is presented in Ref. [6], a new target design for the formation of reverse shocks on GEKKO XII is presented in Ref. [7], and a microscopic study of the formation of radiative shocks on Orion is presented in Ref. [8].

New experimental concepts with laser-driven flows are also introduced. A conceptual design for novel experiments at the SLAC National Accelerator Laboratory ${ }^{[9]}$ looks at the destruction of dust grains in the context of astrophysical shocks, whereas Ref. [10] aims at investigating turbulent hydrodynamic experiments through the TurboHEDP project, with first results at LULI2000 and proposed experiments on OMEGA and NIF.

\section{Experimental diagnostic development}

The development of new diagnostic tools, such as Faraday rotation on OMEGA is presented in Ref. [12], whereas Ref. [11] presents silicon K-shell spectroscopic on SG-II.

\section{Direct astrophysical applications}

A study of high-resolution X-ray spectra of the Vela X-1 binary with the Chandra $\mathrm{X}$-ray observatory, with applications to photoionized plasmas is presented in Ref. [13].

\section{Special issue content}

1. A platform for high-repetition-rate laser experiments on the Large Plasma Device.

2. Using the ROSS optical streak camera as a tool to understand laboratory experiments of laser-driven magnetized shock waves.

3. Analytical modelling of the expansion of a solid obstacle interacting with a radiative shock.

4. Measurement and analysis of K-shell lines of silicon ions in laser plasmas.

5. Laboratory radiative accretion shocks on GEKKO XII laser facility for POLAR project.
6. Analysis of microscopic properties of radiative shock experiments performed at the Orion laser facility.

7. Physical parameter estimation with MCMC from observations of Vela X-1.

8. Generation of strong magnetic fields with a laserdriven coil.

9. Conceptual design of an experiment to study dust destruction by astrophysical shock waves.

10. Experimental platform for the investigation of magnetized-reverse-shock dynamics in the context of POLAR.

11. Turbulent hydrodynamics experiments in high energy density plasmas: scientific case and preliminary results of the TurboHEDP project.

12. Laboratory study of astrophysical collisionless shock at SG-II laser facility.

13. Magnetic reconnection driven by intense lasers.

14. Implementation of a Faraday rotation diagnostic at the OMEGA laser facility.

15. Experimental methods for warm dense matter research.

16. Maser radiation from collisionless shocks: application to astrophysical jets.

\section{Abstracts}

1. A platform for high-repetition-rate laser experiments on the Large Plasma Device

D. B. Schaeffer ${ }^{(\mathrm{a} 1)}$, L. R. Hofer ${ }^{(\mathrm{a} 1)}$, E. N. Knall ${ }^{(\mathrm{a} 1)}$, P. V. Heuer ${ }^{(\mathrm{a} 1)}$, C. G. Constantin ${ }^{(\mathrm{a} 1)}$ and C. Niemann ${ }^{(\mathrm{a} 1)}$

(a1) Department of Physics and Astronomy, University of California - Los Angeles, CA 90095, USA

High Power Laser Science and Engineering, Vol. 6, e17 (2018)

\footnotetext{
Abstract

We present a new experimental platform for studying laboratory astrophysics that combines a high-intensity, highrepetition-rate laser with the Large Plasma Device at the University of California, Los Angeles. To demonstrate the utility of this platform, we show the first results of volumetric, highly repeatable magnetic field and electrostatic potential measurements, along with derived quantities of electric field, charge density and current density, of the interaction between a super-Alfvénic laser-produced plasma and an ambient, magnetized plasma.
} 
2. Using the ROSS optical streak camera as a tool to understand laboratory experiments of laser-driven magnetized shock waves

Andy Liao ${ }^{(\mathrm{a} 1)}$, Patrick Hartigan ${ }^{(\mathrm{a} 1)}$, Gennady Fiksel $^{(\mathrm{a} 2)}$, Brent Blue $^{(\mathrm{a} 3)}$, Peter Graham ${ }^{(\mathrm{a} 4)}$, John Foster ${ }^{(\mathrm{a} 4)}$ and Carolyn Kuranz $^{(\mathrm{a} 2)}$

(a1) Rice University, USA

(a2) University of Michigan - Ann Arbor, USA

(a3) Lawrence Livermore National Laboratory, USA

(a4) Awe Plc, UK

\section{High Power Laser Science and Engineering, Vol. 6, e22} (2018)

\section{Abstract}

Supersonic flows with high Mach number are ubiquitous in astrophysics. High-powered lasers also have the ability to drive high Mach number, radiating shock waves in laboratory plasmas, and recent experiments along these lines have made it possible to recreate analogs of high Mach number astrophysical flows under controlled conditions. Streak cameras such as the Rochester optical streak system (ROSS) are particularly helpful in diagnosing such experiments, because they acquire spatially resolved measurements of the radiating gas continuously over a large time interval, making it easy to observe how any shock waves and ablation fronts present in the system evolve with time. This paper summarizes new ROSS observations of a laboratory analog of the collision of a stellar wind with an ablating planetary atmosphere embedded within a magnetosphere. We find good agreement between the observed ROSS data and numerical models obtained with the FLASH code, but only when the effects of optical depth are properly taken into account.

3. Analytical modelling of the expansion of a solid obstacle interacting with a radiative shock

Th. Michel ${ }^{(\mathrm{a} 1)}$, E. Falize ${ }^{(\mathrm{a} 2)}{ }^{(\mathrm{a} 3)}$, B. Albertazzi ${ }^{(\mathrm{a} 1)}$,

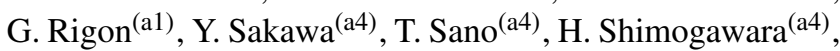

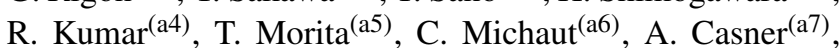

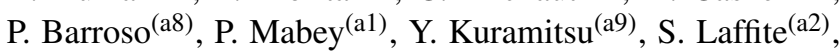
L. Van Box Som ${ }^{(\mathrm{a} 2)(\mathrm{a} 3)(\mathrm{a} 10)}$, G. Gregori( ${ }^{(\mathrm{a} 11)}$, R. Kodama $^{(\mathrm{a} 9)}$,

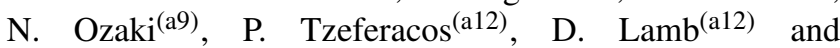
M. Koenig ${ }^{(a 1)(a 9)}$

(a1) LULI - CNRS, École Polytechnique, CEA: Université Paris-Saclay; UPMC Univ Paris 06: Sorbonne Universités F-91128 Palaiseau Cedex, France

(a2) CEA, DAM, DIF, F-91297 Arpajon, France

(a3) CEA Saclay, DSM/Irfu/Service d'Astrophysique, F-91191 Gif-sur-Yvette, France

(a4) Institute of Laser Engineering, Osaka University, Suita, Osaka 565-0871, Japan

(a5) Faculty of Engineering Sciences, Kyushu University, 6-1 Kasuga-Koen, Kasuga, Fukuoka 816-8580, Japan (a6) LUTH, Observatoire de Paris, PSL Research University, CNRS, Université Paris Diderot, Sorbonne Paris Cité, 92190 Meudon, France

(a7) Université de Bordeaux-CNRS-CEA, CELIA, UMR 5107, F-33405 Talence, France

(a8) GEPI, Observatoire de Paris, PSL Research University, CNRS, Université Paris Diderot, Sorbonne Paris Cité, 75014 Paris, France

(a9) Graduate School of Engineering, Osaka University, Suita, Osaka 565-0871, Japan

(a10) LERMA, Observatoire de Paris, PSL Research University, CNRS, Sorbonne Universités, UPMC Univ. Paris 06, F-75005 Paris, France

(a11) Clarendon Laboratory, University of Oxford, Parks Road, Oxford OX1 3PU, UK

(a12) Flash Center for Computational Science, University of Chicago, IL 60637, USA

High Power Laser Science and Engineering, Vol. 6, e30 (2018)

\section{Abstract}

In this paper, we present a model characterizing the interaction of a radiative shock (RS) with a solid material, as described in a recent paper [Koenig et al., Phys. Plasmas, 24, 082707 (2017)], the new model is then related to recent experiments performed on the GEKKO XII laser facility. The RS generated in a xenon gas cell propagates towards a solid obstacle that is ablated by radiation coming from the shock front and the radiative precursor, mimicking processes occurring in astrophysical phenomena. The model presented here calculates the dynamics of the obstacle expansion, which depends on several parameters, notably the geometry and the temperature of the shock. All parameters required for the model have been obtained from experiments. Good agreement between experimental data and the model is found when spherical geometry is taken into account. As a consequence, this model is a useful and easy tool to infer parameters from experimental data (such as the shock temperature), and also to design future experiments.

4. Measurement and analysis of K-shell lines of silicon ions in laser plasmas

Bo Han ${ }^{(\mathrm{a} 1)}$, Feilu Wang ${ }^{(\mathrm{a} 2)}$, Jiayong Zhong ${ }^{(\mathrm{a} 1)}$ (a3), Guiyun Liang $^{(\mathrm{a} 2)}$, Huigang Wei ${ }^{(\mathrm{a} 2)}$, Dawei Yuan ${ }^{(\mathrm{a} 2)}$, Baojun $\mathrm{Zhu}^{(\mathrm{a} 4)}$, Fang $\mathrm{Li}^{(\mathrm{a} 4)}$, Chang $\mathrm{Liu}^{(\mathrm{a} 1)}$, Yanfei $\mathrm{Li}^{(\mathrm{a} 4)}$, Jiarui Zhao $^{(\mathrm{a} 4)}$, Zhe Zhang ${ }^{(\mathrm{a} 4)}$, Chen Wang ${ }^{\left({ }^{(5)}\right)}$, Jun Xiong ${ }^{\left({ }^{(a)}\right)}$, Guo $\mathrm{Jia}^{(\mathrm{a} 5)}$, Neng Hua ${ }^{(\mathrm{a})}$, Jianqiang $\mathrm{Zhu}^{(\mathrm{a} 6)}$, Yutong $\mathrm{Li}^{(\mathrm{a} 4)}{ }^{(\mathrm{a} 3)}$, Gang Zhao ${ }^{(\mathrm{a} 2)}$ and Jie Zhang ${ }^{(\mathrm{a} 7)}$ (a3)

(a1) Department of Astronomy, Beijing Normal University, Beijing 100875, China

(a2) Key Laboratory of Optical Astronomy, National Astronomical Observatories, Chinese Academy of Sciences, Beijing 100012, China

(a3) IFSA Collaborative Innovation Center, Shanghai Jiao Tong University, Shanghai 200240, China 
(a4) National Laboratory for Condensed Matter Physics, Institute of Physics, Chinese Academy of Sciences, Beijing 100190, China

(a5) Research Center for Laser Fusion, China Academy of Engineering Physics, Mianyang 621900, China

(a6) National Laboratory on High Power Laser and Physics, Chinese Academy of Sciences, Shanghai 201800, China

(a7) Key Laboratory for Laser Plasmas (MoE) and Department of Physics and Astronomy, Shanghai Jiao Tong University, Shanghai 200240, China

High Power Laser Science and Engineering, Vol. 6, e31 (2018)

\begin{abstract}
We present laboratory measurement and theoretical analysis of silicon K-shell lines in plasmas produced by Shenguang II laser facility, and discuss the application of line ratios to diagnose the electron density and temperature of laser plasmas. Two types of shots were carried out to interpret silicon plasma spectra under two conditions, and the spectra from $6.6 \AA$ to $6.85 \AA$ were measured. The radiativecollisional code based on the flexible atomic code (RCF) is used to identify the lines, and it also well simulates the experimental spectra. Satellite lines, which are populated by dielectron capture and large radiative decay rate, influence the spectrum profile significantly. Because of the blending of lines, the traditional $G$ value and $R$ value are not applicable in diagnosing electron temperature and density of plasma. We take the contribution of satellite lines into the calculation of line ratios of $\mathrm{He}-\alpha$ lines, and discuss their relations with the electron temperature and density.
\end{abstract}

5. Laboratory radiative accretion shocks on GEKKO XII laser facility for POLAR project

L. Van Bx Som ${ }^{(a 1)}$ (a2) (a3) É. Falize ${ }^{(a 1)}$ (a3), M. Koenig(a4) (a5) Y. Sakawa ${ }^{(26)}$, B. Albertazzi ${ }^{(a 4)}$,

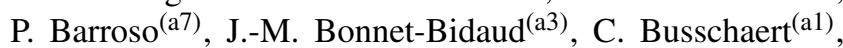

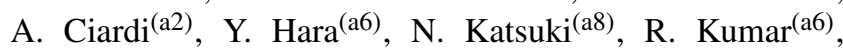

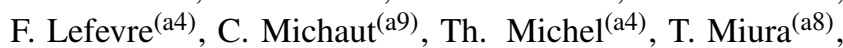

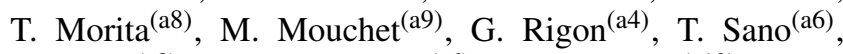

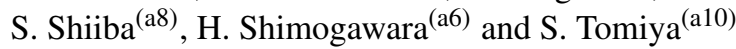

(a1) CEA-DAM-DIF, F-91297 Arpajon, France

(a2) LERMA, Sorbonne Université, Observatoire de Paris, Université PSL, CNRS, F-75005 Paris, France

(a3) CEA Saclay, DSM/Irfu/Service d'Astrophysique, F91191 Gif-sur-Yvette, France

(a4) LULI - CNRS, Ecole Polytechnique, CEA: Université Paris-Saclay; UPMC Univ Paris 06: Sorbonne Université F-91128 Palaiseau Cedex, France

(a5) Graduate School of Engineering, Osaka University, Suita, Osaka 565-0871, Japan

(a6) Institute of Laser Engineering, Osaka University, Suita, Osaka 565-0871, Japan (a7) GEPI, Observatoire de Paris, PSL Research University, CNRS, Université Paris Diderot, Sorbonne Paris Cité, F75014 Paris, France

(a8) Faculty of Engineering Sciences, Kyushu University, 6-1 Kasuga-Koen, Kasuga, Fukuoka 816-8580, Japan

(a) LUTH, Observatoire de Paris, PSL Research University, CNRS, Université Paris Diderot, Sorbonne Paris Cité, F92195 Meudon, France

(a10) Aoyama Gakuin University, Japan

High Power Laser Science and Engineering, Vol. 6, e35 (2018)

\section{Abstract}

A new target design is presented to model high-energy radiative accretion shocks in polars. In this paper, we present the experimental results obtained on the GEKKO XII laser facility for the POLAR project. The experimental results are compared with 2D FCI2 simulations to characterize the dynamics and the structure of plasma flow before and after the collision. The good agreement between simulations and experimental data confirms the formation of a reverse shock where cooling losses start modifying the post-shock region. With the multi-material structure of the target, a hydrodynamic collimation is exhibited and a radiative structure coupled with the reverse shock is highlighted in both experimental data and simulations. The flexibility of the laser energy produced on GEKKO XII allowed us to produce high-velocity flows and study new and interesting radiation hydrodynamic regimes between those obtained on the LULI2000 and Orion laser facilities.

6. Analysis of microscopic properties of radiative shock experiments performed at the Orion laser facility

R. Rodríguez ${ }^{(\mathrm{a} 1)(\mathrm{a} 2)}$, G. Espinosa ${ }^{(\mathrm{a} 1)}$, J. M. Gil ${ }^{(\mathrm{a} 1)}{ }^{(\mathrm{a} 2)}, \mathrm{F}$. Suzuki-Vidal $^{(\mathrm{a} 3)}$, T. Clayson ${ }^{(\mathrm{a} 3)}$, C. Stehlé(a4) and P. Graham ${ }^{(\mathrm{a} 5)}$

(a1) IUNAT, Departamento de Física, Universidad de Las Palmas de Gran Canaria, 35017 Las Palmas de Gran Canaria, Spain

(a2) Instituto de Fusion Nuclear, Universidad Politécnica de Madrid, 28040 Madrid, Spain

(a3) Blackett Laboratory, Imperial College London, London SW7 2AZ, UK

(a4) LERMA, Sorbonne Universités, UPMC, Observatoire de Paris, PSL Research University, CNRS, F-75006 Paris, France

(a5) AWE, Aldermaston, Reading RG7 4PR, UK

High Power Laser Science and Engineering, Vol. 6, e36 (2018)

Abstract
In this work we have conducted a study on the radia-
tive and spectroscopic properties of the radiative precursor 
and the post-shock region from experiments with radiative shocks in xenon performed at the Orion laser facility. The study is based on post-processing of radiationhydrodynamics simulations of the experiment. In particular, we have analyzed the thermodynamic regime of the plasma, the charge state distributions, the monochromatic opacities and emissivities, and the specific intensities for plasma conditions of both regions. The study of the intensities is a useful tool to estimate ranges of electron temperatures present in the xenon plasma in these experiments and the analysis performed of the microscopic properties commented above helps to better understand the intensity spectra. Finally, a theoretical analysis of the possibility of the onset of isobaric thermal instabilities in the post-shock has been made, concluding that the instabilities obtained in the radiative-hydrodynamic simulations could be thermal ones due to strong radiative cooling.

7. Physical parameter estimation with MCMC from observations of Vela X-1

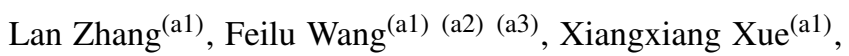
Dawei Yuan ${ }^{(\mathrm{a} 1)}$, Huigang Wei ${ }^{(\mathrm{a} 1)}$ and Gang Zhao ${ }^{(\mathrm{a} 1)(\mathrm{a} 2)}$

(a1) Key Laboratory of Optical Astronomy, National Astronomical Observatories, Chinese Academy of Sciences, Beijing 100012, China

(a2) School of Astronomy and Space Science, University of Chinese Academy of Sciences, Beijing 101408, China

(a3) Graduate School of China Academy of Engineering Physics, Beijing 100196, China

\section{High Power Laser Science and Engineering, Vol. 6, e37 (2018)}

\section{Abstract}

We present a parameter estimate for continua, and He-like triplets of the high resolution X-ray spectra with a Bayesian inference and a Markov Chain Monte Carlo (MCMC) tool. The method is applied for Vela X-1 with three different orbital phases $(\phi)$, Eclipse, $\phi=0.25$, and $\phi=0.5$, which are adopted from the Chandra High-Energy Transmission Grating Spectrometer (HETGS). A parameterized two-component power-law model [Sako et al., Astrophys. J. 525, 921 (1999)] and a multi-Gaussian model are applied to model these continua and He-like triplets, respectively. A uniform distribution over each parameter is used as the prior belief. Posterior probability distribution functions of parameters and the covariances among them are explored by using the MCMC method. The main advantages are (i) all model-based parameters are set to be free instead of artificially fixing some of the parameters during the datamodel fitting; (ii) the contributions from satellite lines are considered; (iii) backgrounds are treated as a correction to the observation errors; and (iv) the confidence interval of each parameter is given. The fitted results show that the column density of scatter component $\left(N_{\mathrm{H}}^{\mathrm{scat}}\right)$ varies from phase to phase, which imply a non-spherical structure of the stellar wind in Vela X-1. Moreover, the wind velocities derived from main lines of each set of He-like triplets show better self-consistency than those in previous publications, which could provide a reliable approach for the diagnostics of photoionized plasma in astrophysical objects and the laboratory.

8. Generation of strong magnetic fields with a laser-driven coil

Zhe Zhang ${ }^{(\mathrm{a} 1)}$, Baojun Zhu ${ }^{(\mathrm{a} 1)(\mathrm{a} 2)}$, Yutong $\mathrm{Li}^{(\mathrm{a} 1)}$ (a2) (a3), Weiman Jiang ${ }^{(\mathrm{a} 1)}$ (a2), Dawei Yuan ${ }^{(\mathrm{a} 4)}$, Huigang $\mathrm{Wei}^{(\mathrm{a} 4)}$,' Guiyun Liang ${ }^{(a 4)}$, Feilu Wang ${ }^{(\mathrm{a} 4)}$, Gang Zhao ${ }^{(\mathrm{a} 4)}$, Jiayong Zhong $^{(\mathrm{a} 3)(\mathrm{a5})}$, Bo Han ${ }^{(\mathrm{a} 5)}$, Neng Hua ${ }^{(\mathrm{a} 6)}$, Baoqiang Zhu ${ }^{(\mathrm{a})}$, Jianqiang Zhu ${ }^{(\mathrm{a})}$, Chen Wang ${ }^{(\mathrm{a})}$, Zhiheng Fang ${ }^{(\mathrm{a7})}$ and Jie Zhang ${ }^{(\mathrm{a} 3)}$ (a8)

(a1) Beijing National Laboratory of Condensed Matter Physics, Institute of Physics, Chinese Academy of Sciences, Beijing 100190, China

(a2) School of Physical Sciences, University of Chinese Academy of Sciences, Beijing 100049, China

(a3) Collaborative Innovation Center of IFSA (CICIFSA), Shanghai Jiao Tong University, Shanghai 200240, China

(a4) Key Laboratory of Optical Astronomy, National Astronomical Observatories, Chinese Academy of Sciences, Beijing 100012, China

(a5) Department of Astronomy, Beijing Normal University, Beijing 100875, China

(a6) National Laboratory on High Power Laser and Physics, Chinese Academy of Sciences, Shanghai 201800, China

(a7) Shanghai Institute of Laser Plasma, Shanghai 201800, China

(a8) Key Laboratory for Laser Plasmas (MoE) and Department of Physics and Astronomy, Shanghai Jiao Tong University, Shanghai 200240, China

High Power Laser Science and Engineering, Vol. 6, e38 (2018)

\section{Abstract}

As a promising new way to generate a controllable strong magnetic field, laser-driven magnetic coils have attracted interest in many research fields. In 2013, a kilotesla level magnetic field was achieved at the Gekko XII laser facility with a capacitor-coil target. A similar approach has been adopted in a number of laboratories, with a variety of targets of different shapes. The peak strength of the magnetic field varies from a few tesla to kilotesla, with different spatiotemporal ranges. The differences are determined by the target geometry and the parameters of the incident laser. Here we present a review of the results of recent experimental studies of laser-driven magnetic field generation, as well as a discussion of the diagnostic techniques required for such rapidly changing magnetic fields. As an extension of the magnetic field generation, some applications are discussed. 
9. Conceptual design of an experiment to study dust destruction by astrophysical shock waves

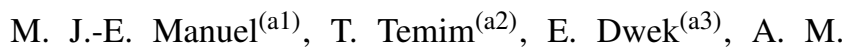
Angulo $^{(\mathrm{a} 4)}$, P. X. Belancourt ${ }^{(\mathrm{a} 4)}$, R. P. Drake ${ }^{(\mathrm{a} 4)}$, C. C. Kuranz $^{(\mathrm{a} 4)}$, M. J. MacDonald ${ }^{(\mathrm{a} 5)}$ and B. A. Remington ${ }^{(\mathrm{a} 6)}$

(a1) General Atomics, 3550 General Atomics Court, San Diego, CA 92121, USA

(a2) Space Telescope Science Institute, 3700 San Martin Drive, Baltimore, MD 21218, USA

(a3) Observational Cosmology Lab, Code 665, NASA Goddard Space Flight Center, Greenbelt, MD 20771, USA

(a4) Climate and Space Science and Engineering, University of Michigan, Ann Arbor, MI 48109, USA

(a5) Department of Physics, University of California, Berkeley, CA 94720, USA

(a6) Lawrence Livermore National Laboratory, Livermore, CA 94450, USA

High Power Laser Science and Engineering, Vol. 6, e39 (2018)

\section{Abstract}

A novel laboratory experimental design is described that will investigate the processing of dust grains in astrophysical shocks. Dust is a ubiquitous ingredient in the interstellar medium (ISM) of galaxies; however, its evolutionary cycle is still poorly understood. Especially shrouded in mystery is the efficiency of grain destruction by astrophysical shocks generated by expanding supernova remnants. While the evolution of these remnants is fairly well understood, the grain destruction efficiency in these shocks is largely unknown. The experiments described herein will fill this knowledge gap by studying the dust destruction efficiencies for shock velocities in the range $\sim 10-30 \mathrm{~km} / \mathrm{s}(\mu \mathrm{m} / \mathrm{ns})$, at which most of the grain destruction and processing in the ISM takes place. The experiments focus on the study of graingrain collisions by accelerating small $(\sim 1 \mu \mathrm{m})$ dust particles into a large ( $\sim 5-10 \mu \mathrm{m}$ diameter) population; this simulates the astrophysical system well in that the more numerous, small grains impact and collide with the large population. Facilities that combine the versatility of high-power optical lasers with the diagnostic capabilities of X-ray free-electron lasers, e.g., the Matter in Extreme Conditions instrument at the SLAC National Accelerator Laboratory, provide an ideal laboratory environment to create and diagnose dust destruction by astrophysically relevant shocks at the micron scale.

10. Experimental platform for the investigation of magnetized-reverse-shock dynamics in the context of POLAR

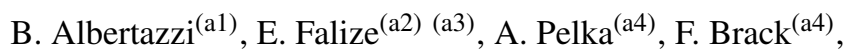

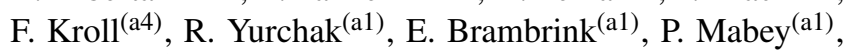

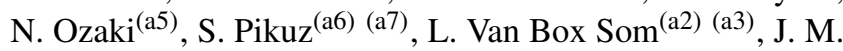

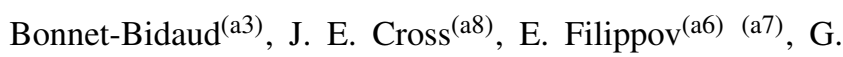

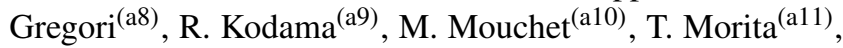
Y. Sakawa ${ }^{(a 9)}$, R. P. Drake ${ }^{(a 12)}$, C. C. Kuranz ${ }^{(\mathrm{a} 12)}$, M. J.-E.

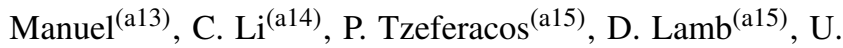
Schramm $^{(\mathrm{a} 4)}$ and M. Koenig(a1) (a5)

(a1) LULI - CNRS, Ecole Polytechnique, CEA: Université Paris-Saclay; UPMC Univ Paris 06: Sorbonne Universités F-91128 Palaiseau Cedex, France

(a2) CEA-DAM-DIF, F-91297 Arpajon, France

(a3) CEA Saclay, DSM/Irfu/Service d'Astrophysique, F91191 Gif-sur-Yvette, France

(a4) Helmholtz-Zentrum Dresden - Rossendorf HZDR, Bautzner Landstraße 400, 01328 Dresden, Germany

(a5) Graduate School of Engineering, Osaka University, Suita, Osaka 565-0871, Japan

(a6) JIHT-RAS, 13-2 Izhorskaya st., Moscow 125412, Russia

(a7) National Research Nuclear University MEPhI, Moscow 115409, Russia

(a8) Department of Physics, University of Oxford, Parks Road, Oxford OX1 3PU, UK

(a9) Institute of Laser Engineering, Osaka University, Suita, Osaka 565-0871, Japan

(a10) LUTH, Observatoire de Paris, UMR CNRS 8102, Université Paris Diderot, 92190 Meudon, France

(a11) Department of Energy Engineering Science, Faculty of Engineering Sciences, Kyushu University, Japan

(a12) Department of Atmospheric, Oceanic, and Space Sciences, University of Michigan, Ann Arbor, MI 48109, USA (a13) General Atomics, San Diego, CA 92121, USA

(a14) Plasma Science and Fusion Center, Massachusetts Institute of Technology, 77 Massachusetts Avenue, Cambridge, MA 02139, USA

(a15) Flash Center for Computational Science, University of Chicago, IL 60637, USA

\section{High Power Laser Science and Engineering Vol. 6, e43 (2018)}

\section{Abstract}

The influence of a strong external magnetic field on the collimation of a high Mach number plasma flow and its collision with a solid obstacle is investigated experimentally and numerically. The laser irradiation $(I \sim 2 \times$ $10^{14} \mathrm{~W} \cdot \mathrm{cm}^{-2}$ ) of a multilayer target generates a shock wave that produces a rear side plasma expanding flow. Immersed in a homogeneous $10 \mathrm{~T}$ external magnetic field, this plasma flow propagates in vacuum and impacts an obstacle located a few $\mathrm{mm}$ from the main target. A reverse shock is then formed with typical velocities of the order of 15-20 $\pm 5 \mathrm{~km} / \mathrm{s}$. The experimental results are compared with $2 \mathrm{D}$ radiative magnetohydrodynamic simulations using the FLASH code. This platform allows investigating the dynamics of reverse shock, mimicking the processes occurring in a cataclysmic variable of polar type. 
11. Turbulent hydrodynamics experiments in high energy density plasmas: scientific case and preliminary results of the TurboHEDP project

A. Casner ${ }^{(\mathrm{a} 1)}$, G. Rigon ${ }^{(\mathrm{a} 2)}{ }^{(\mathrm{a} 3)}$, B. Albertazzi ${ }^{(\mathrm{a} 2)}$, Th.

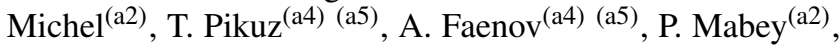
N. Ozaki ${ }^{(\mathrm{a} 6)(\mathrm{a} 7)}$, Y. Sakawa ${ }^{(\mathrm{a} 7)}$, T. Sano ${ }^{(\mathrm{a} 7)}$, J. Ballet ${ }^{(\mathrm{a} 8)}$, P.

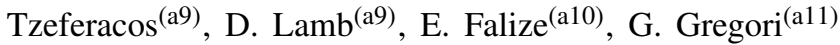
and M. Koenig ${ }^{(\mathrm{a} 2)}$ (a6)

(a1) Université de Bordeaux-CNRS-CEA, CELIA (CEntre Lasers Intenses et Applications), UMR 5107, F-33405 Talence, France

(a2) LULI - CNRS, École Polytechnique, CEA, Université Paris-Saclay, UPMC Univ Paris 06: Sorbonne Universités F-91128 Palaiseau Cedex, France

(a3) ENS Lyon, Université de Lyon, CHELS, 69342 Lyon, France

(a4) Open and Transdisciplinary Research Initiatives, Osaka University, Suita, Osaka 565-0871, Japan

(a5) Joint Institute for High Temperature RAS, Moscow 125412, Russia

(a6) Graduate School of Engineering, Osaka University, Suita, Osaka 565-0871, Japan

(a7) Institute of Laser Engineering, Osaka University, Suita, Osaka 565-0871, Japan

(a8) Laboratoire AIM, CEA-IRFU/CNRS/Université Paris Diderot, Département d'Astrophysique, CEA Saclay, F-91191 Gif sur Yvette, France

(a9) Flash Center for Computational Science, University of Chicago, USA

(a10) CEA, DAM, DIF, F-91297 Arpajon, France

(a11) Clarendon Laboratory, University of Oxford, Parks Road, Oxford OX1 3PU, UK

\section{High Power Laser Science and Engineering, Vol. 6, e44 (2018)}

\section{Abstract}

The physics of compressible turbulence in high energy density (HED) plasmas is an unchartered experimental area. Simulations of compressible and radiative flows relevant for astrophysics rely mainly on subscale parameters. Therefore, we plan to perform turbulent hydrodynamics experiments in HED plasmas (TurboHEDP) in order to improve our understanding of such important phenomena for interest in both communities: laser plasma physics and astrophysics. We will focus on the physics of supernovae remnants which are complex structures subject to fluid instabilities such as the Rayleigh-Taylor and Kelvin-Helmholtz instabilities. The advent of megajoule laser facilities, like the National Ignition Facility and the Laser Megajoule, creates novel opportunities in laboratory astrophysics, as it provides unique platforms to study turbulent mixing flows in HED plasmas. Indeed, the physics requires accelerating targets over larger distances and longer time periods than previously achieved. In a preparatory phase, scaling from experiments at lower laser energies is used to guarantee the performance of future MJ experiments. This subscale experiments allow us to develop experimental skills and numerical tools in this new field of research, and are stepping stones to achieve our objectives on larger laser facilities. We review first in this paper recent advances in high energy density experiments devoted to laboratory astrophysics. Then we describe the necessary steps forward to commission an experimental platform devoted to turbulent hydrodynamics on a megajoule laser facility. Recent novel experimental results acquired on LULI2000, as well as supporting radiative hydrodynamics simulations, are presented. Together with the development of $\mathrm{LiF}$ detectors as transformative $\mathrm{X}$-ray diagnostics, these preliminary results are promising on the way to achieve micrometric spatial resolution in turbulent HED physics experiments in the near future.

12. Laboratory study of astrophysical collisionless shock at SG-II laser facility

Dawei Yuan ${ }^{(\mathrm{a} 1)}$, Huigang Wei ${ }^{(\mathrm{a} 1)}$, Guiyun Liang ${ }^{(\mathrm{a} 1)}$, Feilu Wang ${ }^{(\mathrm{a} 1)}$, Yutong $\mathrm{Li}^{(\mathrm{a} 2)(\mathrm{a} 3)(\mathrm{a} 4)}$, Zhe Zhang ${ }^{(\mathrm{a} 2)}$, Baojun $\mathrm{Zhu}^{(\mathrm{a} 2)}$, Jiarui Zhao ${ }^{(\mathrm{a} 2)}$, Weiman Jiang ${ }^{(\mathrm{a} 2)}$, Bo $\operatorname{Han}^{(\mathrm{a} 5)}$, Xiaoxia Yuan ${ }^{(\mathrm{a} 5)}$, Jiayong Zhong ${ }^{(\mathrm{a} 5)(\mathrm{a} 3)}$, Xiaohui Yuan ${ }^{(\mathrm{a} 6)}$, Changbo $\mathrm{Fu}^{(\mathrm{a} 7)}$, Xiaopeng Zhang ${ }^{(\mathrm{a} 7)}$, Chen Wang ${ }^{(\mathrm{a} 8)}$, Guo $\mathrm{Jia}^{(\mathrm{a} 8)}$, Jun Xiong ${ }^{(\mathrm{a} 8)}$, Zhiheng Fang ${ }^{(\mathrm{a} 8)}$, Shaoen Jiang ${ }^{(\mathrm{a} 9)}$, Kai $\mathrm{Du}^{(\mathrm{a} 9)}$, Yongkun Ding ${ }^{(\mathrm{a} 9)}$, Neng Hua ${ }^{(\mathrm{a} 10)}$, Zhanfeng Qiao $^{(\mathrm{a} 10)}$, Shenlei Zhou ${ }^{(\mathrm{a} 10)}$, Baoqiang Zhu ${ }^{(\mathrm{a} 10)}$, Jianqiang Zhu $^{(\mathrm{a} 10)}$, Gang Zhao ${ }^{(\mathrm{a} 1)(\mathrm{a} 11)}$ and Jie Zhang ${ }^{(\mathrm{a} 6)(\mathrm{a} 3)}$

(a1) Key Laboratory of Optical Astronomy, National Astronomical Observatories, Chinese Academy of Sciences, Beijing 100012, China

(a2) National Laboratory for Condensed Matter Physics, Institute of Physics, Chinese Academy of Sciences, Beijing 100190, China

(a3) Collaborative Innovation Center of IFSA (CICIFSA), Shanghai Jiao Tong University, Shanghai 200240, China

(a4) School of Physical Sciences, University of Chinese Academy of Sciences, Beijing 100049, China

(a5) Department of Astronomy, Beijing Normal University, Beijing 100875, China

(a6) Key Laboratory for Laser Plasmas (MoE) and Department of Physics and Astronomy, Shanghai Jiao Tong University, Shanghai 200240, China

(a7) INPAC and School of Physics and Astronomy, Shanghai Jiao Tong University, Shanghai Key Laboratory for Particle Physics and Cosmology, Shanghai 200240, China

(a8) Shanghai Institute of Laser Plasma, Shanghai 201800, China

(a9) Research Center for Laser Fusion, China Academy of Engineering Physics, Mianyang 621900, China

(a10) National Laboratory on High Power Laser and Physics, Chinese Academy of Sciences, Shanghai 201800, China

(a11) School of Astronomy and Space Science, University of Chinese Academy of Sciences, Beijing 101408, China 
High Power Laser Science and Engineering, Vol. 6, e45 (2018)

\begin{abstract}
Astrophysical collisionless shocks are amazing phenomena in space and astrophysical plasmas, where supersonic flows generate electromagnetic fields through instabilities and particles can be accelerated to high energy cosmic rays. Until now, understanding these micro-processes is still a challenge despite rich astrophysical observation data have been obtained. Laboratory astrophysics, a new route to study the astrophysics, allows us to investigate them at similar extreme physical conditions in laboratory. Here we will review the recent progress of the collisionless shock experiments performed at SG-II laser facility in China. The evolution of the electrostatic shocks and Weibel-type/filamentation instabilities are observed. Inspired by the configurations of the counter-streaming plasma flows, we also carry out a novel plasma collider to generate energetic neutrons relevant to the astrophysical nuclear reactions.
\end{abstract}

\section{Magnetic reconnection driven by intense lasers}

Jiayong Zhong ${ }^{(\mathrm{a} 1)(\mathrm{a} 2)}$, Xiaoxia Yuan ${ }^{(\mathrm{a} 1)}$, Bo Han ${ }^{(\mathrm{a} 1)}$, Wei Sun ${ }^{(\mathrm{a} 1)}$ and Yongli Ping ${ }^{(\mathrm{a} 1)}$

(a1) Department of Astronomy, Beijing Normal University, Beijing 100875, China

(a2) IFSA Collaborative Innovation Center, Shanghai Jiao Tong University, Shanghai 200240, China

High Power Laser Science and Engineering, Vol. 6, e48 (2018)

\begin{abstract}
Laser-driven magnetic reconnection (LDMR) occurring with self-generated B fields has been experimentally and theoretically studied extensively, where strong B fields of more than megagauss are spontaneously generated in highpower laser-plasma interactions, which are located on the target surface and produced by non-parallel temperature and density gradients of expanding plasmas. For properties of the short-lived and strong B fields in laser plasmas, LDMR opened up a new territory in a parameter regime that has never been exploited before. Here we review the recent results of LDMR taking place in both high and low plasma beta environments. We aim to understand the basic physics processes of magnetic reconnection, such as particle accelerations, scale of the diffusion region, and guide field effects. Some applications of experimental results are also given especially for space and solar plasmas.
\end{abstract}

14. Implementation of a Faraday rotation diagnostic at the OMEGA laser facility

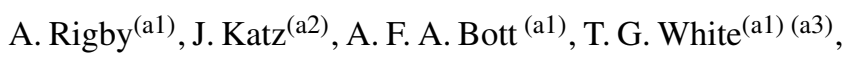
P. Tzeferacos ${ }^{(\mathrm{a} 1)(\mathrm{a} 4)}$, D. Q. Lamb ${ }^{(\mathrm{a} 4)}$, D. H. Froula ${ }^{(\mathrm{a} 2)}$ and G. Gregori $^{(a 1)(a 4)}$

(a1) Department of Physics, University of Oxford, Parks Road, Oxford OX1 3PU, UK

(a2) Laboratory for Laser Energetics, University of Rochester, 250 E. River Rd, Rochester, NY 14623, USA

(a3) Department of Physics, University of Nevada, Reno, NV89557, USA

(a4) Department of Astronomy and Astrophysics, University of Chicago, 5640 S. Ellis Ave, Chicago, IL 60637, USA

High Power Laser Science and Engineering, Vol. 6, e49 (2018)

\section{Abstract}

Magnetic field measurements in turbulent plasmas are often difficult to perform. Here we show that for $\gg k G$ magnetic fields, a time-resolved Faraday rotation measurement can be made at the OMEGA laser facility. This diagnostic has been implemented using the Thomson scattering probe beam and the resultant path-integrated magnetic field has been compared with that of proton radiography. Accurate measurement of magnetic fields is essential for satisfying the scientific goals of many current laser-plasma experiments.

15. Experimental methods for warm dense matter research Katerina Falk ${ }^{(\mathrm{a} 1)(\mathrm{a} 2)}$

(a1) Helmholtz-Zentrum Dresden-Rossendorf, Bautzner Landstrasse 400, 01328 Dresden, Germany

(a2) Institute of Physics of the ASCR, Na Slovance 1999/2, 18221 Prague, Czech Republic

High Power Laser Science and Engineering, Vol. 6, e59 (2018)

\section{Abstract}

The study of structure, thermodynamic state, equation of state (EOS) and transport properties of warm dense matter (WDM) has become one of the key aspects of laboratory astrophysics. This field has demonstrated its importance especially concerning the internal structure of planets, but also other astrophysical bodies such as brown dwarfs, crusts of old stars or white dwarf stars. There has been a rapid increase in interest and activity in this field over the last two decades owing to many technological advances including not only the commissioning of high energy optical laser systems, z-pinches and X-ray free electron lasers, but also short-pulse laser facilities capable of generation of novel particle and $\mathrm{X}$-ray sources. Many new diagnostic methods have been developed recently to study WDM in its full complexity. Even ultrafast nonequilibrium dynamics has been accessed for the first time thanks to subpicosecond laser pulses achieved at new facilities. Recent years saw a number of major discoveries with direct implications to astrophysics such as the formation of diamond at pressures relevant to interiors of frozen giant planets like Neptune, metallic hydrogen under 
conditions such as those found inside Jupiter's dynamo or formation of lonsdaleite crystals under extreme pressures during asteroid impacts on celestial bodies. This paper provides a broad review of the most recent experimental work carried out in this field with a special focus on the methods used. All typical schemes used to produce WDM are discussed in detail. Most of the diagnostic techniques recently established to probe WDM are also described. This paper also provides an overview of the most prominent examples of these methods used in experiments. Even though the main emphasis of the publication is experimental work focused on laboratory astrophysics primarily at laser facilities, a brief outline of other methods such as dynamic compression with z-pinches and static compression using diamond anvil cells (DAC) is also included. Some relevant theoretical and computational efforts related to WDM and astrophysics are mentioned in this review.

16. Maser radiation from collisionless shocks: application to astrophysical jets

D. C. Speirs ${ }^{(\mathrm{a} 1)}$, K. Ronald ${ }^{(\mathrm{a} 1)}$, A. D. R. Phelps ${ }^{(\mathrm{a} 1)}$, M. E. $\mathrm{Koepke}^{(\mathrm{a} 2)}$, R. A. Cairns ${ }^{(\mathrm{a} 3)}$, A. Rigby ${ }^{(\mathrm{a} 4)}, \mathrm{F}_{\mathrm{C}} \mathrm{Cruz}^{(\mathrm{a} 5)}, \mathrm{R}$. Trines $^{(\mathrm{a} 6)}$, R. Bamford ${ }^{(\mathrm{a} 6)}$, B. J. Kellett ${ }^{(\mathrm{a})}$, B. Albertazzi ${ }^{(\mathrm{a} 7)}$ J. E. $\operatorname{Cross}^{(\mathrm{a})}$, F. Fraschetti ${ }^{(\mathrm{a} 9)}$, P. Graham ${ }^{(\mathrm{a} 10)}$, P. M. Kozlowski $^{(\mathrm{a} 8)}$, Y. Kuramitsu ${ }^{(\mathrm{a} 11)}$, F. Miniati ${ }^{(\mathrm{a} 8)}$, T. Morita ${ }^{(\mathrm{a} 12)}$, M. Oliver ${ }^{(\mathrm{a} 8)}$, B. Reville ${ }^{(\mathrm{a} 13)}$, Y. Sakawa $^{(\mathrm{a} 12)}$, S. Sarkar ${ }^{(\mathrm{a} 8)}$, C.

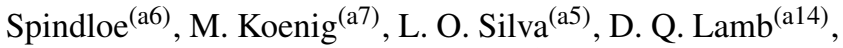
P. Tzeferacos ${ }^{(\mathrm{a} 8)(\mathrm{a} 14)}$, S. Lebedev ${ }^{(\mathrm{a} 15)}$, G. Gregori ${ }^{(\mathrm{a} 8)(\mathrm{a} 14)}$ and R. Bingham ${ }^{(\mathrm{a} 1)(\mathrm{a} 6)}$

(a1) Department of Physics, SUPA, University of Strathclyde, Glasgow, Scotland, G4 0NG, UK

(a2) Department of Physics, West Virginia University, Morgantown, WV 26506-6315, USA

(a3) School of Mathematics and Statistics, University of St. Andrews, Fife, Scotland, KY16 9SS, UK

(a4) Department of Physics, University of Oxford, Parks Road, Oxford, OX1 3PU, UK

(a5) GoLP/Instituto de Plasmas e Fusãu Nuclear, Instituto Superior Técnico, Universidade de Lisboa, 1049-001 Lisbon, Portugal

(a6) STFC Rutherford Appleton Laboratory, Chilton, Didcot, Oxon, England, OX11 0QX, UK

(a7) Laboratoire pour l'Utilisation de Lasers Intenses, UMR7605, CNRS CEA, Université Paris VI Ecole Polytechnique, 91128 Palaiseau Cedex, France

(a8) Department of Physics, University of Oxford, Parks Road, Oxford, OX1 3PU, UK

(a9) Departments of Planetary Sciences and Astronomy, University of Arizona, Tucson, AZ 85721, USA

(a10) AWE, Aldermaston, Reading, West Berkshire RG7 4PR, UK

(a11) Department of Physics, National Central University, Taoyuan 320, Taiwan

(a12) Institute of Laser Engineering, Osaka University, 2-6 Yamadaoka, Suita, Osaka 565-0871, Japan (a13) School of Mathematics and Physics, Queens University Belfast, Belfast BT7 1NN, UK

(a14) Department of Astronomy and Astrophysics, University of Chicago, 5640 S. Ellis Ave, Chicago, IL 60637, USA

(a15) Imperial College London, London, SW72AZ, UK

High Power Laser Science and Engineering, Vol. 7, e17 (2019)

\section{Abstract}

This paper describes a model of electron energization and cyclotron-maser emission applicable to astrophysical magnetized collisionless shocks. It is motivated by the work of Begelman, Ergun and Rees [Astrophys. J. 625, 51 (2005)] who argued that the cyclotron-maser instability occurs in localized magnetized collisionless shocks such as those expected in blazar jets. We report on recent research carried out to investigate electron acceleration at collisionless shocks and maser radiation associated with the accelerated electrons. We describe how electrons accelerated by lower-hybrid waves at collisionless shocks generate cyclotron-maser radiation when the accelerated electrons move into regions of stronger magnetic fields. The electrons are accelerated along the magnetic field and magnetically compressed leading to the formation of an electron velocity distribution having a horseshoe shape due to conservation of the electron magnetic moment. Under certain conditions the horseshoe electron velocity distribution function is unstable to the cyclotron-maser instability [Bingham and Cairns, Phys. Plasmas 7, 3089 (2000); Melrose, Rev. Mod. Plasma Phys. 1, 5 (2017)].

\section{References}

1. D. B. Schaeffer, L. R. Hofer, E. N. Knall, P. V. Heuer, C. G. Constantin, and C. Niemann, High Power Laser Sci. Eng. 6, e17 (2018).

2. A. Liao, P. Hartigan, G. Fiksel, B. Blue, P. Graham, J. Foster, and C. Kuranz, High Power Laser Sci. Eng. 6, e22 (2018).

3. B. Albertazzi, E. Falize, A. Pelka, F. Brack, F. Kroll, R. Yurchak, E. Brambrink, P. Mabey, N. Ozaki, S. Pikuz, L. Van Box Som, J. M. Bonnet-Bidaud, J. E. Cross, E. Filippov, G. Gregori, R. Kodama, M. Mouchet, T. Morita, Y. Sakawa, R. P. Drake, C. C. Kuranz, M. J.-E. Manuel, C. Li, P. Tzeferacos, D. Lamb, U. Schramm, and M. Koenig, High Power Laser Sci. Eng. 6, e43 (2018).

4. D. Yuan, H. Wei, G. Liang, F. Wang, Y. Li, Z. Zhang, B. Zhu, J. Zhao, W. Jiang, B. Han, X. Yuan, J. Zhong, X. Yuan, C. Fu, X. Zhang, C. Wang, G. Jia, J. Xiong, Z. Fang, S. Jiang, K. Du, Y. Ding, N. Hua, Z. Qiao, S. Zhou, B. Zhu, J. Zhu, G. Zhao, and J. Zhang, High Power Laser Sci. Eng. 6, e45 (2018).

5. D. C. Speirs, K. Ronald, A. D. R. Phelps, M. E. Koepke, R. A. Cairns, A. Rigby, F. Cruz, R. Trines, R. Bamford, B. J. Kellett, B. Albertazzi, J. E. Cross, F. Fraschetti, P. Graham, P. M. Kozlowski, Y. Kuramitsu, F. Miniati, T. Morita, M. Oliver, B. Reville, Y. Sakawa, S. Sarkar, C. Spindloe, M. Koenig, L. O. Silva, D. Q. Lamb, P. Tzeferacos, S. Lebedev, G. Gregori, and R. Bingham, High Power Laser Sci. Eng. 7, e17 (2019). 
6. Th. Michel, E. Falize, B. Albertazzi, G. Rigon, Y. Sakawa, T. Sano, H. Shimogawara, R. Kumar, T. Morita, C. Michaut, A. Casner, P. Barroso, P. Mabey, Y. Kuramitsu, S. Laffite, L. Van Box Som, G. Gregori, R. Kodama, N. Ozaki, P. Tzeferacos, D. Lamb, and M. Koenig, High Power Laser Sci. Eng. 6, e30 (2018).

7. L. Van Box Som, É. Falize, M. Koenig, Y. Sakawa, B. Albertazzi, P. Barroso, J.-M. Bonnet-Bidaud, C. Busschaert, A. Ciardi, Y. Hara, N. Katsuki, R. Kumar, F. Lefevre, C. Michaut, Th. Michel, T. Miura, T. Morita, M. Mouchet, G. Rigon, T. Sano, S. Shiiba, H. Shimogawara, and S. Tomiya, High Power Laser Sci. Eng. 6, e35 (2018).

8. R. Rodríguez, G. Espinosa, J. M. Gil, F. Suzuki-Vidal, T. Clayson, C. Stehlé, and P. Graham, High Power Laser Sci. Eng. 6, e36 (2018).

9. M. J.-E. Manuel, T. Temim, E. Dwek, A. M. Angulo, P. X. Belancourt, R. P. Drake, C. C. Kuranz, M. J. MacDonald, and B. A. Remington, High Power Laser Sci. Eng. 6, e39 (2018).
10. A. Casner, G. Rigon, B. Albertazzi, Th. Michel, T. Pikuz, A. Faenov, P. Mabey, N. Ozaki, Y. Sakawa, T. Sano, J. Ballet, P. Tzeferacos, D. Lamb, E. Falize, G. Gregori, and M. Koenig, High Power Laser Sci. Eng. 6, e44 (2018).

11. B. Han, F. Wang, J. Zhong, G. Liang, H. Wei, D. Yuan, B. Zhu, F. Li, C. Liu, Y. Li, J. Zhao, Z. Zhang, C. Wang, J. Xiong, G. Jia, N. Hua, J. Zhu, Y. Li, G. Zhao, and J. Zhang, High Power Laser Sci. Eng. 6, e31 (2018).

12. A. Rigby, J. Katz, A. F. A. Bott, T. G. White, P. Tzeferacos, D. Q. Lamb, D. H. Froula, and G. Gregori, High Power Laser Sci. Eng. 6, e49 (2018).

13. L. Zhang, F. Wang, X. Xue, D. Yuan, H. Wei, and G. Zhao, High Power Laser Sci. Eng. 6, e37 (2018).

14. Z. Zhang, B. Zhu, Y. Li, W. Jiang, D. Yuan, H. Wei, G. Liang, F. Wang, G. Zhao, J. Zhong, B. Han, N. Hua, B. Zhu, J. Zhu, C. Wang, Z. Fang, and J. Zhang, High Power Laser Sci. Eng. 6, e38 (2018).

15. J. Zhong, X. Yuan, B. Han, W. Sun, and Y. Ping, High Power Laser Sci. Eng. 6, e48 (2018).

16. K. Falk, High Power Laser Sci. Eng. 6, e59 (2018). 